\title{
Defensa del realismo metafísico en la obra de Sir Karl R. Popper
}

\section{Defense of metaphysical realism in the work of Sir Karl R. Popper}

Received: December 3, 2020

\section{Resumen}

La aparición de la mecánica cuántica en la primera mitad del Siglo XX significó un enorme avance en la ciencia y en particular en la física, con una irradiación sobre todo el quehacer de las comunidades científicas del mundo. Ha sido una de las teorías más exitosas de la historia del pensamiento científico. No obstante, sus problemas de interpretación fueron y son muy complejos. Sir Karl. R. Popper intento defender la postura del "Realismo Metafísico" ante este verdadero tsunami, producido por la teoría cuántica. En este texto revisamos esa situación llevada adelante por Popper frente a la interpretación cuántica del mundo. Por su parte, el objetivo de este artículo dice relación con explorar la importancia del realismo metafísico en el quehacer de la ciencia. Mientras que la metodología utilizada, dirá relación con tomar un gran hecho científico, como fue el caso de la teoría cuántica, y llevar adelante un análisis en torno a las diversas interpretaciones de la misma que implican, finalmente la discusión sobre el realismo metafísico como un supuesto que da sentido al trabajo científico.

Palabras Clave: Conocimiento Científico, Mecánica Cuántica, Realidad, Realismo Metafísico, Verdad.
Accepted: February 11, 2021

Written by:

Mario Lagomarsino-Montoya ${ }^{8}$ https://orcid.org/0000-0001-9204-4745

Juan Guillermo Estay-Sepúlveda9 https://orcid.org/0000-0001-7348-5529

Juan Mansilla-Sepúlveda ${ }^{10}$ https://orcid.org/0000-0001-8175-7475

Alex Véliz-Burgos ${ }^{11}$ https://orcid.org/0000-0003-1371-9041

Héctor Salazar-Cayuleo ${ }^{12}$ https://orcid.org/0000-0001-6084-5185

\begin{abstract}
After the appearance of quantum mechanics in the first half of the twentieth century, wich meant a huge advance in science and particularly in physics, with a radiation above all the task of the scientific communities of the world. It has been one of the most successful theories in the history of scientific thought. However, their problems of interpretation were and are very complex. Sir Karl R. Popper attempted to defend the position of the "metaphysical realism" in the face of this true Tsunami, produced by quantum theory. In this text, we review this situation carried out by Popper in front of the quantum interpretation of the world. For its part, the objective of this article is related to exploring the importance of metaphysical realism in the work of science. While the methodology used would be related to taking a great scientific fact, as was the case with quantum theory, and carrying out an analysis around the various interpretations of it that they imply, finally the discussion about metaphysical realism as a assumption that gives meaning to scientific work.
\end{abstract}

Key Words: Scientific knowledge, Quantum Mechanics, Reality, Metaphysical Realism, Truth.

\footnotetext{
${ }^{8}$ Investigador Universidad Adventista de Chile, Chile.

${ }^{9}$ Investigador Universidad Católica de Temuco, Chile y Universidad Adventista de Chile, Chile. Universidad de Salamanca, España.

${ }^{10}$ Decano e Investigador de la Universidad Católica de Temuco, Chile.

11 Académico e investigador Universidad de Los Lagos, Chile.

12 Académico e investigador Universidad Adventista de Chile, Chile.
} 


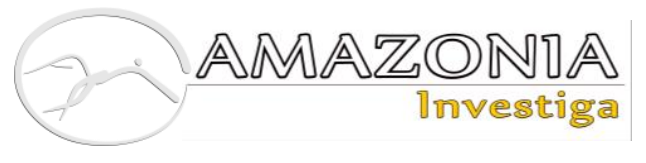

\section{Introducción}

El desarrollo de este artículo tiene su génesis en lo pensado y obrado por el filósofo austriaco en la década del noventa del siglo pasado, en ese sentido, se encuentra en la perspectiva de lo que Karl Popper entendía por el realismo metafísico y su importancia en el desarrollo de las ciencias y de la epistemología sobre las que se trabajára, para efectos de su comprensión y metodologías. No obstante, en esta versión iremos planteando las observaciones que a la postura realista metafísica se le pueden efectuar con el tiempo transcurrido y principalmente con la aparición de tecnologías que al menos han generado las denominadas "Realidades Múltiples", para no ser acusados de defender un "Realismo Ingenuo". El objetivo de esta ingación dice relación con explorer la importancia que asigna Popper al "Realismo Metafísico" como supuesto para el trabajo de la ciencia, mientras que la metodología utilizada dira relación con analizar un gran acontecimiento cietífico, como es la aparición de la teoría cuántica en el Siglo $\mathrm{XX}$, comparando las diversas interpretaciones que tuvo la misma y como en esa diatribe surge la discusión sobre el realism metafíco y su impacto en la investigación científica. Por su parte, los resultados esperados están circunscritos a los de una investigación metafísica - epistemológica y que en consecuencia se traducen en argumentaciones, esta vez, en favor o contra de la postura del realismo sostenida por Sir Karl Popper.

\section{Desarrollo}

\section{El Realismo Metafísico}

El realismo es la postura que sostiene que el mundo existe, tal como lo vemos, independiente del sujeto que lo observa a través de sus sentidos. Tal es la tesis que Karl Popper ha defendido en su obra y a la cual ha dedicado gran parte de la misma. ¿Pero es el realismo una tesis que merezca ser defendida? ¿No es algo tan obvio que al parecer no necesitará mayor defensa?

Estas interrogantes se irán respondiendo a medida que vayamos desarrollando el problema de la "variable Realidad" en la ciencia contemporánea y de la misma forma el esfuerzo realizado por Popper por mostrar lo coherente, fundamental e incluso trivial del realismo. Lo anterior apunta vectorialmente en contra del generalizado clima de animadversión hacia este postulado metafísico. El tema central es la existencia de la realidad que plantea el realismo.
Así Rosental y Ludin (1970: 327) definen realidad como "aquello que realmente existe y se desarrolla, contiene en él mismo su propia esencia y sus propias leyes, así como los resultados de su propia acción y desarrollo. Tal realidad es la realidad objetiva en toda su concreción".

Ahora bien, históricamente se ha definido el realismo metafísico de formas diferentes. De esta manera, Putnam (1994: 22) en Las Mil Caras del Realismo sostiene que el realismo metafísico se puede resumir en las siguientes tesis "1.- El mundo existe independientemente de nuestros conceptos y representaciones 2.Ese mundo objetivo e independiente tiene una estructura determinada; es decir, está compuesto de cosas, propiedades, hechos objetivos que existen independientemente de que nosotros los conozcamos o no. 3.- Una representación verdadera o correcta del mundo es aquella que se refiere a objetos realmente existentes en el mundo y describe las propiedades que esos objetos realmente tienen. 4.- Existe (aunque no la conozcamos todavía) una imagen o representación completa del mundo objetivo tal como es en sí mismo". Igualmente, Díaz (2007: 185) señala: "A quienes consideran que el mundo realmente existe más allá de nuestro conocimiento de él se los llama realistas. El realismo del sentido común cree que el mundo es tal como lo percibimos. El realista científico, por su parte, no sólo cree que el mundo existe, sino también que el conocimiento científico es como un espejo que refleja el mundo. Un biólogo realista cree que el virus es una entidad real que responde con exactitud a la definición científica".

Por su parte, Grayling (1982: 233) sostiene que: "Realismo es la tesis de que el mundo está determinadamente constituido, esto es, que tiene su carácter independientemente de toda experiencia o conocimiento de él, de modo que las sentencias acerca del mundo son verdaderas o falsas". Por último, veamos sobre el realismo metafísico, lo que nos dice Hacking (1983: 67) cuando sostiene que las entidades, estados y procesos descritos por las teorías correctas en realidad existen. Los protones, fotones, campos de fuerza y hoyos negros son tan reales como las uñas de los pies, turbinas o volcanes".

El diseño para exponer esta situación y posterior propuesta de Popper será sumariamente la siguiente. En primer término, exponer cuándo y 
con qué o quién surge el problema de la realidad en la ciencia contemporánea, mientras que en segundo término señalar las consideraciones con las que Popper intenta enfrentar esta problemática y de paso destacar la enorme importancia que para la ciencia, la epistemología e incluso la moral, tiene este postulado de carácter metafísico.

Ahora bien, la defensa del realismo llevada adelante por este epistemólogo, también se conecta con lo que se ha denominado la objetividad en la ciencia y del conocimiento científico. En este marco, y en una notación introductoria a Teoría Cuántica y el Cisma en Física, Popper (1992 b: 22) mantiene: "Realismo es el mensaje de este libro. Está vinculado con la objetividad, también en la teoría de la probabilidad. Este vínculo da lugar a la interpretación propensivista. El realismo está vinculado con el racionalismo, con la realidad de la mente humana, de la creatividad y del sufrimiento humano".

A continuación, veremos sinópticamente cómo es que el problema de la "Realidad" se generó en la ciencia del siglo XX y con ello la perdida de importancia del realismo. Ulteriormente, notaremos los esfuerzos de Popper para retrotraer esta situación.

\section{Situación Revolucionaria en la Ciencia Contemporánea a partir del surgimiento de la Mecánica Cuántica}

En correspondencia con el orden recién estipulado, pasaremos a exponer cómo surge “El Problema de la Realidad" en la ciencia contemporánea y en consecuencia el ingreso con tanta energía del observador y por lo mismo de las posturas subjetivistas en epistemología. A pesar que es con la mecánica cuántica (de ahora en adelante MC) con que el problema de realidad emerge de forma contundente, el resquebrajamiento de este postulado tiene algunos antecedentes preliminares, entre los cuales se encuentran algunos de los siguientes: la aparición de una filosofía subjetivista o idealista, la adopción de una interpretación instrumentalista de las teorías científicas y el surgimiento de la física probabilística, con la equivocada interpretación que la probabilidad (en el sentido del cálculo de probabilidades) mide la carencia de conocimiento de un sujeto que lleva adelante mediciones. No obstante, lo anterior (aunque al parecer fuese lo mismo) es con la aparición de la MC (y sus problemas de interpretación) con que el problema de la realidad se pondrá de manifiesto en la ciencia contemporánea, causando con ello una revolución paradigmática de enormes consecuencias en la ciencia, cuyos efectos llegan incluso al día de hoy, pleno siglo XXI.

La MC fue y es una teoría muy exitosa -si pudiéramos hacer una trayectoria de los aportes de la MC a las tecnologías del siglo XX y XXI son enormes y permanentes. Tal vez los más importantes que ninguna ciencia haya entregado antes- en lo que dice relación con su aparataje instrumental y predictivo. Sin embargo, la misma presenta graves problemas de interpretación. En esta línea argumental, el físico teórico Selleri (1986: 111) ha señalado que es "razonable decir que nadie entiende realmente por qué funciona la teoría cuántica, $\mathrm{y}$ que su epistemología está todavía muy atrasada respecto de la física".

En torno a la interpretación de la MC se producirá una gran controversia en el siglo $\mathrm{XX}$ con una enorme irradiación hasta nuestros días. La mencionada controversia se desarrollará con la participación de los físicos más destacados de la primera mitad del siglo pasado. $\mathrm{Y}$ en una parte sustancial, la disputa se centrará en torno a la situación de la Realidad y en consecuencia a la postura sostenida por el Realismo Metafísico. En la histórica formulación de los teóricos cuánticos, sustentadores de la interpretación de la Escuela de Copenhague, es muy clara la postura de abandono del realismo y por consiguiente, la admisión de una epistemología idealista, subjetivista, instrumentalista (y por lo mismo eminentemente pragmática que apunta a consideraciones de predicción; sin mayor preocupación por avanzar hacia aspectos de compresión).

En este contexto, se pueden apreciar las siguientes formulaciones. Heisenberg (1986: 14-15) afirma: "La noción de realidad objetiva $[\ldots]$ se ha disuelto $[\ldots]$ en la transparente claridad de una matemática que describe, no el comportamiento de las partículas elementales, pero si nuestro conocimiento de dicho comportamiento".

Por otra parte, Bohr citado por Bell (1991: 201) declara: "No existe un Mundo cuántico. Hay sólo una descripción cuántica abstracta. Es equivocado pensar que la tarea de la física consiste en descubrir cómo es la naturaleza. La física se ocupa de lo que podemos decir acerca de la naturaleza". En la misma dirección, Wheeler citado por Fernández (2018: 314) afirma: "Ningún fenómeno es un fenómeno real hasta que es un fenómeno observado". 
De este modo, podemos apreciar la entrada del subjetivismo y del observador en la ciencia contemporánea y por lo mismo del rechazo del realismo, esto es, de la existencia objetiva de la realidad, del mundo. Lo anterior, una existencia independiente del sujeto (y de sus estados de conciencia).

Siguiendo la argumentación, de los destacados científicos citados, se hace depender la existencia del mundo de la presencia de un observador o sujeto cognoscente. Pero si hacemos depender la existencia del mundo de la presencia del observador esto lleva necesariamente a la tesis de que el mundo objetivo no puede existir sin la mente de un observador que le asigna soporte ontológico. Las consecuencias epistemológicas de tales posturas, advertidas también por otros estudiosos y filósofos de las ciencias, son de una complejidad enorme para la ciencia, pues entre otras consecuencias la ciencia se quedaría sin referente a quien estudiar; sin realidad.

Asimismo, Ortoli y Pharabod mantienen que el hacer depende de la existencia del mundo (de la realidad) de la presencia de un observador, y tiene las siguientes consecuencias: "Semejante posición puede conducirnos a consideraciones por lo menos inquietantes: el mundo material no existiría independientemente del observador" (Ortoli y Pharabod, 1985: 11). Esta sentencia es bastante potente, desde el punto de vista epistemológico, y desde luego que es una posición que reafirma el realismo metafísico, defendido por nuestro autor.

Ahora bien, en la misma línea argumental Armstrong (1966: 73) sustenta: "Admitir, que las impresiones o datos no pueden existir sin una mente que los tenga, ello plantea en seguida un problema a la doctrina de que el mundo físico no es más que nuestras impresiones sensoriales de él. Porque cuando nadie está percibiendo un objeto físico determinado, nadie está teniendo impresiones sensoriales de este objeto. Parece, pues, que [... se] está obligado a decir que es lógicamente imposible que los objetos existan impercibidos; que la noción de existencia física impercibida carece de sentido".

En consecuencia, será el problema de la realidad, el que tendrá Popper en vista cuando emprende su reacción en defensa del realismo metafísico y de la objetividad de la ciencia, dos conceptos epistemológicos muy difíciles de defender en el siglo XXI. No obstante, de enorme importancia para el conocimiento científico y su progreso dialéctico. La hipótesis popperiana puede ser enunciada de la siguiente manera: la ciencia trata sobre el mundo real el cual existe objetivamente, es decir, independientemente de si es o no percibido por un observador. Las teorías de la ciencia se ocupan de un mundo real y van a ser "verdaderas" o "falsas" en la medida y audacia en que intenten describir, explicar y comprender el mundo.

En la visión que mantiene Popper sobre el desarrollo y avance de la ciencia, ésta progresa a través de teorías especulativas, de presunciones, de soluciones tentativas para nuestros problemas (que se denominan conjeturas) las cuales son sometidas a los más fuertes controles en el proceso de contrastación con la realidad. Controles guiados por la actitud crítica del científico, ya sea en el terreno o el laboratorio. Mediante la crítica podemos llegar a establecer en qué están erradas nuestras teorías o, entre otras palabras, en qué no describen adecuadamente el mundo, a saber, la Realidad. Las teorías no son solamente formalismos matemáticos para hacer predicciones de eventos; en donde suelen ser muy buenos instrumentos. Son, al fin y al cabo, herramientas con las que intentamos conocer el mundo, el universo; la realidad en última instancia.

La defensa del Realismo metafísico y de la objetividad de la ciencia, por parte de Popper, se hace mayormente inteligible cuando advertimos las tesis cargadas de idealismo y subjetivismo por parte de los más grandes físicos cuánticos del siglo XX: Heisenberg, Bohr, Jordan, Wheeler y Heitler, entre otros. Pero en esta defensa del realismo y de la objetividad de la ciencia, nuestro autor no estuvo sólo. Por el contrario, se mantuvo en una buena compañía epistemológica. En defensa del realismo metafísico se han pronunciado científicos de tantos o más méritos, que los anteriormente mencionados. Bell (1991: 161) sostiene que "yo encuentro provechosa la idea de que existe un mundo real, y que nuestra tarea es tratar de descubrir cosas sobre él, y que la técnica para hacer eso es ciertamente construir modelos y ver cuán lejos nos llevan en la descripción del mundo real". Así Einstein (1983: 74) declara: "La creencia en un mundo externo independiente del sujeto perceptor, es la base de toda la ciencia natural". Por otro lado, el descubridor del efecto fotoeléctrico argumenta: "Yo (creo) en un mundo de algo que tiene una existencia objetiva que yo trato de capturar mediante teorías osadamente especulativas" (Paty, 1979: 52). De igual forma, 
Bohm, ante la pregunta de si él cree que el mundo externo existe objetivamente, afirma: "Todos los físicos lo creen así. Por ejemplo, hablan del universo como habiendo evolucionado desde antes de que no existiera nadie para observarlo. Ahora bien, salvo que se le atribuya a Dios como lo hizo el Obispo Berkeley (y la mayoría de los físicos no desean hacerlo), uno es incapaz de resolver el problema de cómo es que existe el universo sin físicos para observarlo, o sin alguien más que lo observe" (Davies y Brown, 1989: 161).

Por otra parte, d'Espagnat (1980) advirtiendo de las consecuencias de abandonar el realismo, sostiene que la "ciencia quedaría reducida a un recetario para predecir las observaciones futuras a partir de las ya realizadas. Cualquier noción de ciencia como 'el estudio de la naturaleza' sería imposible. La naturaleza pasaría a ser una ilusión" (d'Espagnat, 1980: 85).

\section{La Defensa del Realismo Metafísico en la Obra de Karl. R. Popper}

Una vez que hemos contextualizado el problema del realismo en la ciencia contemporánea, pasaremos a continuación a la defensa de este postulado que se ha efectuado desde la filosofía de las ciencias y la epistemología. En concordancia con lo anterior, auscultaremos qué importancia atribuye Popper al realismo en su obra y luego, en un grupo de ocho tesis, apreciaremos la magnitud de la importancia que nuestro autor le asigna a este postulado en el desarrollo de la ciencia y del conocimiento científico. A continuación, el propio epistemólogo señala: "La cuestión central aquí es el realismo. Es decir, la realidad del mundo físico en que vivimos: el hecho de que este mundo existe con independencia de nosotros; que existió antes de que existiese la vida según nuestras mejores hipótesis, y que continuará existiendo, por lo que sabemos, mucho después que todos nosotros hayamos desaparecido" (Popper, 1992: 67). El realismo es, en la obra de Popper, un supuesto metafísico, esto es, indemostrable y a la vez infalsable. A diferencia de lo que ocurre con las teorías científicas, que, en términos reales, podrían estar sujetas a una falsación material. Es oportuno recordar que dentro de los aportes efectuados por Karl. R. Popper a la Ciencia y a la epistemología se encuentra el criterio de "falsabilidad", el cual dice relación con que las teorías científicas para tener el estatus de tales deben tener un conjunto no vacío de falsadores potenciales. Lo anterior, como un criterio metodológico permanente. Para lo anterior, ver "La Lógica de la Investigación Científica"
(1977), "Realismo y el Objetivo de la Ciencia" (1986). Es interesante la crítica de Karl Otto Apel formula al criterio de "falsabilidad", propuesto por Popper (Apel, 1991). Así cuando, confiesa su realismo lo hace de la siguiente manera: "Creo que vivimos en un mundo real un mundo que presenta cierto tipo de orden estructural que se nos aparece bajo la forma de leyes" (Popper, 1977: 120).

Pero antes de continuar, dejemos establecido el tipo de realismo sustentado en esta obra. Se defiende un realismo metafísico-ontológico, que tiene que ver finalmente con aquello que hay o que existe, más allá de las puras impresiones sensoriales. Por consiguiente, el realismo respaldado es aquel que sustenta que tanto el mundo, así como los objetos, existen fuera e independientes de la conciencia, de un sujeto o de un observador eventual. Ahora bien, el propio Einstein no veía ningún peligro en aceptar la existencia objetiva y autónoma de los objetos reales. Junto a las estructuras espaciotemporales que corresponden. Popper, coincidiendo con Einstein, va un poco más allá y sostiene que algo, un objeto, puede ser denominado "real", si y sólo si, puede recibir una patada y en principio ese objeto pueda devolverla, ya sea como una irradiación del golpe aplicado sobre él mismo.

Al ser un realista metafísico, Popper mantiene que las teorías científicas tratan sobre el mundo real. En este sentido ha afirmado que "soy realista porque sostengo que el problema de si son verdaderas o no las teorías hechas por el hombre depende de los hechos reales, los cuales no son en absoluto un producto humano [...] Las teorías hechas por el hombre pueden chocar con esos hechos reales, por lo que en nuestra búsqueda de la verdad podemos vernos obligados a reajustar nuestras teorías o abandonarlas" (Popper, 1992 a: 296). La importancia de este realismo para la ciencia la veremos a continuación. No obstante, existe otro ámbito, tal vez el más importante, en el cual se ha defendido el realismo metafísico. Ese ámbito está vinculado al terreno de lo moral y será con el que concluiremos este texto.

No obstante ser el realismo un supuesto metafísico y de no pertenecer a la conocida metodología popperiana, tiene sin embargo una importancia decisiva, como veremos a continuación en las siguientes ocho tesis, que van en defensa de la coherencia y necesidad del postulado para la propia racionalidad del progreso científico. 
Primera Tesis: El realismo metafísico nos permite presumir que discutimos (o que los científicos discuten) acerca de problemas reales, es decir, problemas que nos afectan a todos. El realismo no soluciona un problema eliminándolo, sino que intenta darle explicación a través de potentes teorías que intentan indagar sobre la realidad.

Segunda Tesis: Popper identifica la tesis opuesta al realismo como aquella que subyace $o$ que subyació en la ciencia contemporánea. La denomina como 'Idealismo': "Todo lo real o todo lo que existe es lo que puede ser percibido por un sujeto experiencial o un observador". Ahora bien, para Popper el que todo conocimiento se derive primariamente de los sentidos lleva necesariamente a la concepción de que todo conocimiento tiene que ser de origen de nuestra experiencia sensorial actual o pasada. Así sostiene: "Sobre esta base subjetiva, no puede construirse ninguna teoría objetiva: el mundo viene a ser la totalidad de mis ideas, de mis sueños. La doctrina de que el mundo es un sueño -es decir el idealismo- es irrefutable" (Popper, 1992 c: 63). Cuando la ciencia asume una epistemología idealista, como es el caso de la interpretación de Copenhague, se está produciendo una seria confusión entre dos aspectos del conocimiento (Siguiente Tesis). La confusión de estos aspectos tiene la consecuencia inmediata y necesaria para la práctica científica dice relación con lo siguiente: se confunde el proceso de contrastación de una teoría científica con su referente. Este relevante punto que ha sido destacado por Popper, también ha sido advertido por Bunge (1978: 15), quien afirma que "mientras que para contrastar nuestras ideas son menester experiencias de varias clases, éstas no constituyen las referentes de aquéllas. El referente propuesto de cualquier idea física es una cosa real". En esta misma línea Papp (1979: 42) señaló que "la investigación científica es una incursión en lo desconocido, que a menudo conduce a hallazgos más sorprendentes que los buscados".

Tercera Tesis: Popper ha señalado que la confusión, que acabamos de apreciar (Segunda Tesis), entre la contrastación y el referente de una teoría es solamente una pequeña parte del gran embrollo que significa adoptar una epistemología idealista como sucedió en la ciencia contemporánea (del siglo XX y con seguidores hoy). La aceptación del realismo metafísico y no del idealismo, nos permite hacer una separación entre dos aspectos implicados en el conocimiento científico. Por un lado, el aspecto ontológico y por el otro el aspecto epistemológico. Lo ontológico no depende de lo que conozcamos, de lo que creamos ni de lo que observemos. Esto último dice relación con los métodos de decisión que nos permiten conocer, es decir, el aspecto epistemológico. Por el contrario, lo que hay o lo que existe depende de su existencia objetiva, y en modo alguno tiene que ver con el conocimiento, la creencia o lo que un sujeto observa. Un hecho que permite darnos cuenta de lo anterior es la refutación que pueden sufrir nuestras ideas acerca de la realidad; la refutación de nuestras teorías. De esta manera, Popper (1992 b: 27) señala que alguna "teorías son tan arriesgadas que pueden chocar con la realidad: son las teorías contrastables de la ciencia. Y cuando chocan, entonces sabemos que hay una realidad: algo que puede informarnos de que nuestras ideas son erróneas. Y, por eso, el realista tiene razón".

Cuarta Tesis: El realismo metafísico es el que permite explicar tanto la coincidencia como las diferencias en los reportes que obtienen los científicos del medio. Esta capacidad explicativa del realismo también puede ser usado como un argumento en su apoyo ¿por qué son semejantes las observaciones de dos científicos? Desde la perspectiva idealista, esta pregunta es difícil de responder, aunque seguramente se podría responder de forma $a d$ hoc. En cambio, desde el prisma realista, se puede expresar muy fácilmente (fácilmente = racionalmente): ambos científicos encuentran semejanzas en sus reportes sensoriales porque los dos están observando "un mismo estado de cosas", que existe en forma independiente y objetiva. En este punto, tenemos que efectuar una necesaria distinción. Por un lado, el argumento esbozado es muy fuerte en favor del realismo y por otro el científico necesita postular, por motivos de explicación -si la "Explicación" en la ciencia es importante es un tema en discusión hoy en la contemporánea epistemología. En nuestra postura y recogiendo las posturas de Popper, en toda su obra, la explicación y la comprensión de los "sucesos", "hechos" y "realidad", son el gran tema de la ciencia y del conocimiento científico-, la existencia de un universo material que existe objetivamente. En cuanto apoyo del realismo podemos citar, en este momento, la argumentación de Rosenblueth (1988: 55), quien declara que aceptamos el postulado del realismo metafísico porque "aun cuando tenemos conciencia de que podemos evocar o dirigir voluntariamente algunos de nuestros procesos mentales, hay otros, aquellos cuyo origen atribuimos a los objetos o eventos 
materiales, que no podemos ni evocar ni suprimir voluntariamente". En tanto que es un postulado fundamental para la ciencia, dejemos que nuevamente Rosenbluet ilustre este punto. Así señala que "es necesario postular la existencia de un universo material externo para explicar satisfactoriamente algunos de nuestros propios procesos conscientes" y que "existen procesos conscientes en algunas de las otras entidades que forman parte del universo material, para poder explicar, entender muchos aspectos de su comportamiento", agregando que "la existencia de este universo material para explicar algunas recurrencias $\mathrm{O}$ regularidades que aparecen en nuestras sensaciones o percepciones" (Rosenblueth, 1988: 58). Concluimos que el postulado realista es fundamental para la ciencia y a la vez esa situación constituye en fuerte argumento para su razón de ser.

Quinta Tesis: Uno de los testimonios, que ha sostenido Popper en favor del realismo está vinculado con el tipo de explicación científica, que ha esgrimido de su clásica obra de 1934 (Popper, 1977). En este argumento, el realismo se encuentra asociado a la idea de descubrimiento, mediante la noción de evidencia independiente. Popper respalda que el objetivo de la ciencia es encontrar "explicaciones" a los distintos problemas que se intentan remediar (explicar, comprender y luego intentar hacer predicciones). En la argumentación científica, tratamos de "explicar un problema" ("explicandum" o "explanandum") intentando descubrir sus premisas o condiciones antecedentes ("explicans" o “explanans") (Nagel, 1978 y Hempel, 1988). De igual manera, nunca podremos estar seguros de que una explicación sea verdadera, pero si le podemos exigir que haya evidencia independiente en su apoyo que pueda ser contrastada; no tan solamente con el problema que intenta explicar. Dejemos que el propio Popper, establezca la relación entre evidencia independiente, descubrimiento $\mathrm{y}$ realismo. Así nuestro autor afirma que "la tarea de la ciencia, que como he sugerido, es encontrar explicaciones satisfactorias, difícilmente puede entenderse si no somos realistas. Porque una explicación satisfactoria es una que no es ad hoc; y esta idea -la de evidencia independiente- difícilmente puede entenderse sin la idea de descubrimiento, de progreso hacia niveles más profundos de explicación; sin la idea, por tanto, de que hay algo que tenemos que descubrir; y algo que discutir críticamente" (Popper, 1986: 185-186).
Sexta Tesis: El realismo metafísico se encuentra vinculado a la racionalidad de la ciencia. Entenderemos "racionalidad de la ciencia" en dos sentidos:

a) En un primer sentido, el realismo está conexo con la racionalidad de la ciencia, puesto que la creencia en un mundo externo hace aparecer racional la búsqueda que intenta el científico, en cuanto a lograr explicaciones a problemas con los cuales se enfrenta. De esta manera, ante la interrogante del sentido común: ¿Qué hacen los científicos en su laboratorio, terreno o despacho?, podemos responder desde la perspectiva realista. Desde la óptica realista, podemos entender que el científico (Ya sea aquel que se dedica a las ciencias naturales o aquel que se dedica a las ciencias sociales. No hay ninguna diferencia entre ambos. Particularmente en lo referido a la "Unidad de Método". Queremos decir "Método Científico"), invente teorías y que las someta a severas contrastaciones, y que muchas veces esas teorías resulten equivocadas y que en consecuencia en la dialéctica del método científico deba generar otras en su ánimo y disposición de seguir explicando y sondeando la desconocida realidad. Se usa el concepto de "dialéctica" muy a pesar de Popper. Pero realmente es un movimiento dialectico, la propuesta de funcionamiento que él hace del método científico. Lo de la "teoría única", será una crítica que Popper recibirá, por parte de Imre Lakatos, La Metodología de los Programas de Investigación Científica (Lakatos, 1988).

b) En un segundo sentido, el realismo está conexo con la racionalidad de la ciencia en cuanto se dice que ésta persigue como meta la verdad. La ciencia busca la verdad, más allá de los buenos pronósticos y predicciones que hacen las teorías, convertidas en impresionantes aparatos matemáticos de gran precisión; no obstante, de sentido común se espera la comprensión de los sucesos, hechos y la verdad sobre la realidad, por muy complejo que resulte encontrar la verdad. El realismo metafísico entrega sentido y hace aparecer racional la búsqueda de la verdad en la ciencia y de esta manera nos hace entender ciertas afirmaciones epistemológicas tales como: a pesar de que lo buscado es la verdad, nunca podremos estar seguros de que una teoría finalmente se encuentre: "verificada", "justificada" o que es "verdadera". La búsqueda de la verdad es racional en la ciencia, pues, nos lleva a 
construir medios para lograr ciertos fines. Lo anterior, ya que la indagación por la verdad nos lleva a construir teorías, la cuales, van a ser verdaderas o falsas en la medida cómo describan el mundo físico objetivo. Las teorías serán verdaderas o falsas según correspondan a los hechos que intentan describir y explicar. Lo últimamente expuesto da sentido y hace racional la búsqueda de la verdad, ya que nos dice, que la verdad no está en nosotros ni en supuestos estados de conciencia, sino que tenemos que buscarla fuera; en la realidad objetiva que se intenta describir y explicar.

Séptima Tesis: Si el realismo está en lo cierto, entonces eso explicaría la razón por la cual nuestro conocimiento puede ser solamente conjetural. Esta argumentación se puede manifestar de la siguiente manera: es mucho lo que conocemos, pero nuestra ignorancia es infinita. La tesis realista es la que mejor nos permite una vieja interrogante: ¿Por qué no hemos descubierto o encontrado la teoría verdadera del mundo? Si el mundo fuera nuestra creación lo conoceríamos $\mathrm{y}$, por consiguiente, podríamos dar cuenta de él, en una única teoría verdadera establecida para siempre. Pero como tenemos evidencia, constantemente la realidad está desechando nuestras mejores teorías y obligándonos a sondear mejor en su misteriosa y desconocida composición. Esa ha sido la tarea de los científicos por el trascurso de los siglos, respetando opiniones como la de Feyerabend (2000). Así la propuesta realista sustenta que no hemos encontrado la teoría verdadera, porque el mundo y el universo) se encuentra en una suerte de cambio continuo y en cualquier momento nuestras mejores teorías, pueden quedar refutadas o ser simplemente una descripción insuficiente de la realidad que se intenta conocer. Este punto lo ha definido Popper de la siguiente manera: "Si el realismo es verdadero, nuestra creencia en la realidad del mundo y en las leyes físicas no puede ser demostrable, no puede mostrarse que es seguro o 'razonable' por medio de ningún razonamiento valido. En otras palabras, si el realismo está en lo cierto, no podemos esperar tener más que un conocimiento conjetural: el milagro es, más bien, que hayamos tenido tanto éxito en nuestra búsqueda de conjeturas. Y al decir esto, no tengo en mente sólo los milagrosos éxitos de los últimos trescientos años" (Popper, 1983: 143).

De lo anterior se desprenden las siguientes consecuencias: a) No podemos establecer una teoría como 'justificada' o 'verdadera' para siempre;

b) Todas las teorías se mantienen solamente como conjeturas, por muy bien corroboradas que estén en un momento ' $\boldsymbol{t}$ '.

c) Aprendemos solamente de la detección de los errores, de las teorías que se logran falsar a través de las contrastaciones críticas.

Octava Tesis: Uno de los grandes esfuerzos de Popper fue haber combatido contra toda forma de subjetivismo en la ciencia. Esto se debe a que nuestro autor considera que el subjetivismo (y la entrada ontológica del observador que auspicia) es el responsable del rechazo del realismo en varios ámbitos. Entre ellos el ontológico, en la objetividad de la ciencia, en lo epistemológico y en una cierta interpretación del cálculo de probabilidades. Así contra el subjetivismo, no se ha tan sólo defendido el realismo sino también el objetivismo o la objetividad en la ciencia y en la teoría del conocimiento. Por consiguiente, la defensa del objetivismo puede ser apreciada en por lo menos cinco sentidos:

a) Objetividad Ontológica: La defensa del realismo puede ser entendida como una importante aproximación a la tesis objetivista en el plano ontológico.

b) Objetividad de los Enunciados científicos: Contrastación intersubejtiva: Históricamente, desde 1934, e incluso en una obra anterior, (pero publicada posterior a esa fecha) (Popper, 1998), Popper ha defendido un tipo de objetividad que tiene que ver con los enunciados de la ciencia. La objetividad de la ciencia y de sus enunciados radica en el hecho que pueden ser contrastados intersubjetivamente. Ningún sentimiento de convicción puede justificar un enunciado científico por sí sólo.

c) Objetividad de la Ciencia: critica intersubjetiva: Esta generalización que realiza Popper, desde la contrastación intersubjetiva, le lleva a señalar que la objetividad de la ciencia radica en la crítica mutua entre los distintos científicos, en el marco de una "Comunidad Científica" (Kuhn 1993) en una suerte de confrontación hostil-amistosa.

d) Conocimiento Objetivo: la Tesis de los Tres Mundos: Popper ha sostenido la existencia de un "Mundo 3", que se constituye con el surgimiento de un lenguaje descriptivo, junto al mundo físico "Mundo 1" y al mundo de nuestros estados 
de conciencia y disposiciones, "Mundo 2". Ha desarrollado esta postura (metafísica) haciendo una distinción entre el contenido semántico y objetivo de las proposiciones y su conformación sintáctica. De este modo, al "Mundo 3" sólo pertenecen los contenidos de las proposiciones, es decir: teorías, hipótesis científicas, argumentos, los problemas y las soluciones intentadas, los puntos de discusión, la matemática, la lógica, los algoritmos, entre otros elementos generados por los hombres, pero que han llegado alcanzar una existencia objetiva de los sujetos; incluso de su creador. El conocimiento científico que discurre sobre un mundo que existe objetivamente, pertenece al "Mundo 3". Allí puede ser estudiado, descifrado y se pueden seguir haciendo de forma infinita descubrimientos sobre él mismo; se ha convertido en un conocimiento en sí mismo que ha superado a su creador; en lo relativo a las consecuencias e implicaciones lógicas que él mismo tiene. Aunque el "Mundo 3" es una creación de la actividad pensante del hombre, sin embargo, genera su propia legalidad y autonomía; una autonomía dinámica y en constante relación. No se trata de una autonomía inmutable y petra, como es el mundo de las ideas de Platón, por ejemplo.

e) Objetividad del Cálculo de Probabilidades: de Frecuencias a Propensiones: Popper sostiene que el cálculo de probabilidades mide propiedades de ciertos sistemas físicos de las disposiciones experimentales. La probabilidad no es una medida de nuestro conocimiento imperfecto o de nuestra ignorancia respecto de lo que ocurre en los sistemas físicos. Siempre dentro de las interpretaciones objetivas, Popper ha avanzado desde una interpretación frecuencial a una interpretación propensivista del cálculo de probabilidades. Considera objetivas a ambas interpretaciones. Ahora su inclinación por la propensivista el propio autor lo declara: "conjeturo que (las) propensiones son físicamente reales en el mismo sentido que las fuerzas de atracción o repulsión, por ejemplo, pueden ser físicamente reales. Estimo ahora que la interpretación frecuencial es un intento de prescindir de la realidad física oculta" (Popper, 1992 d: 30-31). Concluiremos la defensa del objetivismo en Popper citando el trabajo de su compatriota, el epistemólogo, Paul Weingartner, quien infieriendo una regla desde la lectura popperiana, sostiene: "Mantente lejos del subjetivismo. Evita los conceptos subjetivos en la formación de (las) hipótesis científicas. La enorme importancia de esta regla resulta manifiesta en todos los campos científicos: el intento del fenomenalismo de sustituir todas las expresiones objetivas de la forma 'en el punto espacio-temporal $\mathrm{K}$ acontece esto $\mathrm{y}$ esto' por las subjetivas 'en el punto espacio-temporal $\mathrm{K}$ el observador $\mathrm{A}$ experimenta esto y esto' ha fracasado teóricamente y, en la práctica, no se llevó a cabo en ninguna ciencia, pues en el resultado científico debe hacerse abstracción del lado subjetivo: para la ciencia es importante lo que es el caso, no lo que el observador le parece que sea el caso" (Weingartner, 1992: 28).

De esta manera, hemos revisado la defensa del realismo metafísico en la obra de Sir Karl R. Popper, contextualizando con la problemática de la ciencia del siglo XX e intentando mostrar el intento de solución que nuestro autor propuso desde el enfoque de un realista metafísico. Para cerrar esta exposición, es oportuno notar que Popper no solamente defendió el realismo, para las temáticas propias de la ciencia y la epistemología, sino también desde un punto de vista moral. Enfoque que tiene que ver con una concepción del hombre, su sufrimiento y dolor real. En concordancia, Popper se refiere a uno de los acontecimientos más trágicos de la historia de la humanidad, ocurrido en el siglo $\mathrm{XX}$ : "He argüido en favor del realismo en varios lugares. Mis argumentos son en parte racionales, en parte ad hominem y en parte, incluso éticos. Me parece que el ataque al realismo, aunque intelectualmente interesante e importante, es bastante inaceptable, sobre todo después de dos guerras mundiales y del sufrimiento real -sufrimiento evitable- que produjeron inexcusablemente; y me parece que cualquier argumento contra el realismo que se base en la moderna teoría atómica -en la mecánica cuántica- debe ser silenciado por el recuerdo de la realidad de los acontecimientos de Hiroshima y Nagasaki (Digo esto lleno de admiración por la teoría cuántica y por la mecánica cuántica modernas y por los científicos que han trabajado y trabajan en ese campo)" (Popper, 1992 b: 26).

\section{Conclusión}

La defensa del "Realismo Metafísico", tiene que ver con una posición tremendamente de sentido común, la cual dice relación con la 
existencia independiente de la realidad de un sujeto percipiente.

Para motivos de la ciencia y del conocimiento científico el postulado que establece el "Realismo Metafísico" es muy importante toda vez que despeja y aclara la relación entre el aspecto ontológico y epistemológico en la investigación. Las percepciones no son la realidad.

Desde varios puntos de vistas se hace necesaria la defensa del realismo. Ya hemos hablado de los aspectos involucrados en el conocimiento científico. Pero también, es necesario señalar los aspectos morales de la postura. No son nuestras percepciones las que sufren. Son Sujetos concretos, los que pasan grandes penurias y desgracias. En consecuencia, cuando trabajamos sobre la realidad, estamos trabajando sobre objetos, entes y seres humanos que existen de forma independiente "a mis", "a nuestros" estados de conciencia y/o percepciones.

\section{Agradadecimientos}

Los Autores - Investigadores, desean agradecer el trabajo laborioso y muy comprometido de la Señorita Karla Pérez Jara, estudiante de tercer año de la carrera de Administración Pública de la Universidad de Valparaíso, Chile. La Señorita Pérez Jara, presenta muy buenas condiciones para la investigación y, tal vez lo más importante, una enorme entusiasmo por impulsar el trabajo que conlleva a la generación de conocimiento científico y en consecuencia a la diatriba posterior que perfecciona éste.

\section{Referencias}

Armstrong, D. (1966) La Percepción y el Mundo Físico. Madrid: Editorial Tecnos.

Apel, K.O. (1991). Teoría de la Verdad y Ética del Discurso. Barcelona: Editorial Paidos.

Bell, J. (1991) Lo Decible y lo Indecible en Mecánica Cuántica. Madrid: Editorial Alianza Universidad.

Bunge, M. (1978) Filosofía de la Física. Barcelona: Editorial Ariel.

Davies, P, \& Brown, J. (1989) El Espíritu en el Átomo. Una Discusión sobre los misterios de la Física Cuántica. Madrid: Editorial Alianza.

D’Espagnat, B. (1980) Teoría Cuántica y Realidad en selecciones de Scientific American. Ed. H. Blume.

Díaz, E. (2007). Metodología de las Ciencias Sociales. Buenos Aires: Editorial Biblos.
Einstein, A. (1983) Sobre la Teoría de la Relatividad. Madrid: Editorial Sarpe.

Fernández, P. (2018) Ciencia y Filosofía: Aspectos Ontológicos y Epistemológicos de la Ciencia Contemporánea. Madrid: Editorial Caligrama.

Feyerabend, P. (2000) Tratado Contra el Método. Madrid: Editorial Tecnos.

Grayling, G. (1982). An Introduction to Philosophical Logic. Sussex, The Press Limited.

Hacking, I. (1983). Representanting and Intervening: introductory in the Philosophy of Natural Science. Cambridge University Press.

Heisenberg, W. (1986) La Imagen de la Naturaleza en la Física Actual. Buenos Aires: diciones Orbis.

Kuhn, T.H. (1993) La Estructura de las Revoluciones Científicas. Santiago: Editorial FCE.

Lakatos, I. (1988) La Metodología de los Programas de Investigación Científica. Madrid: Editorial Alianza.

Hempel, C. (1988) La Explicación Científica. Estudios sobre la Filosofía de las Ciencias. Barcelona: Editorial Paidos.

Nagel, E. (1978) La Estructura de la Ciencia. Problemas de la Lógica de la Investigación Científica. Barcelona: Editorial Paidos.

Ortoli, S., \& Pharabod, J.P. (1985) El Cantico de la Cuántica ¿Existe el Mundo? Barcelona: Editorial Gedisa.

Papp, D. (1979) Ideas Revolucionarias en la Ciencia. Santiago: Editorial Universitaria.

Paty, M. (1979) Einstein en la Tempestad. En Deligeorge, S. (1990) El Mundo Cuántico, Madrid: Editorial Alianza Universidad.

Popper, K.R. (1977) La Lógica de la Investigación Científica. Madrid: Editorial Tecnos.

Popper, K.R. (1983) Conjeturas y Refutaciones. El Desarrollo del Conocimiento Científico. Barcelona: Editorial Paídos.

Popper, K.R. (1986) Realismo y el Objetivo de la Ciencia. Madrid: Editorial Tecnos.

Popper, K.R. (1992a) Conocimiento Objetivo. Un Enfoque Evolucionista. 342. $4^{\mathrm{a}}$ edición. Madrid: Editorial Tecnos.

Popper, K.R. (1992b) Teoría Cuántica y el Cisma en Física. Madrid: Editorial Tecnos.

Popper, K.R. (1992c) El Universo Abierto. Un Argumento en Favor del Indeterminismo. Madrid: Editorial Tecnos.

Popper, K.R. (1992 d) Un Mundo de Propensiones. Madrid: Editorial Tecnos.

Popper, K.R. (1998) Los Dos Problemas Fundamentales de la Epistemología. Basado en los Manuscritos de los años 1930 - 1933. Madrid: Editorial Tecnos. 
Putnam, H. (1994) Las Mil Caras del Realismo. Barcelona: Editorial Paidos.

Rosenblueth, A. (1988) Mente y Cerebro. México: Editorial Siglo XXI.

Rosental, M., \& Ludin, P. (1970) Diccionario Filosófico. Barcelona: Editorial Book Trade.
Selleri, F. (1986) El debate de la mecánica cuántica. Madrid: Alianza Editorial.

Weingartner, P. (1992) La Filosofía de Karl Popper en Cinco Principios, en Karl Popper. Filósofo de la Racionalidad Crítica. Santiago: Editorial Pontificia Universidad Católica de Chile. 